\title{
O papel mediador da autoeficácia na relação entre a sobrecarga de trabalho e as dimensões de Burnout em professores
}

\author{
Mary Sandra Carlotto - Pontificia Universidade Católica- Rio Grande do Sul, Porto Alegre, Brasil \\ Sofia Raquel da Silva Dias - Centro Hospitalar Vila Nova de Gaia, Espinho, Portugal \\ Jaqueline Brito Vidal Batista - Universidade Federal da Paraíba, João Pessoa, Brasil \\ Liciane Diehl - Centro Universitário UNIVATES, Lajeado, Brasil
}

\begin{abstract}
Resumo
O objetivo do presente estudo foi avaliar se a autoeficácia funciona como variável mediadora na relação entre a sobrecarga de trabalho e as dimensões de burnout. Participaram da pesquisa 982 professores de escolas públicas e privadas. Foram utilizados como instrumentos de pesquisa o questionário para avaliação da síndrome de burnout, versão para professores, a escala geral de autoeficácia e a subescala de sobrecarga laboral da organizational Stress Questionnaire. Os resultados obtidos por meio da análise de regressão linear confirmaram a hipótese de que a autoeficácia desempenha um papel mediador entre a sobrecarga de trabalho e as dimensões de burnout. O resultado aponta para a importância de intervenções que contemplem o desenvolvimento da autoeficácia em professores como medida de prevenção ou reabilitação da síndrome de burnout.

Palavras-chave: síndrome de burnout; sobrecarga laboral; autoeficácia, professores; mediação.
\end{abstract}

The mediational role of self-efficacy in the relation between workload and burnout' dimensions in teachers

\begin{abstract}
The objective of this study was to evaluate if self-efficacy can be a mediating variable in the relationship between workload and the dimensions of burnout. The research involved 982 teachers from public and private schools. The Questionnaire for the assessment of burnout Syndrome was used as research instrument, a version for teachers, the General Scale of Self-efficacy and the Subscale of Workload the Organizational Stress Questionnaire. The results obtained by means of linear regression analysis confirmed the hypothesis that self-efficacy plays a mediating role between workload and the dimensions of burnout. The result demonstrates the importance of interventions directed to the development of self-efficacy in teachers as a measure of prevention or rehabilitation of the burnout syndrome.

Keywords: burnout syndrome; mediation; teachers; self-efficacy; workload.
\end{abstract}

El papel mediador de la autoeficacia en la relación entre la sobrecarga de trabajo y las dimensiones de burnout en profesores

\begin{abstract}
Resumen
El objetivo del estudio fue evaluar si la autoeficacia funciona como variable mediadora en la relación entre la sobrecarga de trabajo y las dimensiones de burnout. Participaron 982 profesores de escuelas públicas y privadas. Se utilizaron como herramientas el Cuestionario para Evaluación del Síndrome de burnout, versión para profesores, la Escala General de Autoeficacia y la Sub-escala de Sobrecarga Laboral de la Organizational Stress Questionnaire. Los resultados obtenidos mediante el análisis de regresión linear confirmaron la hipótesis de que la autoeficacia ejerce un papel mediador entre la sobrecarga de trabajo y las dimensiones de burnout. El resultado muestra la importancia de intervenciones que contemplen el desarrollo de la autoeficacia en profesores, como medida de prevención o rehabilitación del Síndrome burnout.

Palabras-clave: síndrome de burnout; sobrecarga laboral; autoeficacia; profesores; mediación.
\end{abstract}

\section{Introdução}

A Organização Internacional do Trabalho (OIT, 2012) aponta que o estresse ocupacional é, na atualidade, uma das mais importantes questões de saúde mundial e tem sido alvo de preocupação em muitos países e nos mais diferentes contextos de trabalho. Tal preocupação se deve ao impacto negativo que causa na saúde física e mental dos trabalhadores, assim, elevando os já altos índices de afastamento laboral, com evidente diminuição da produtividade nas organizações de trabalho. Estressores ocupacionais, de acordo com Guglielmi e
Tatrow (1998), quando persistentes, podem ocasionar a síndrome de burnout (SB). Essa síndrome é o resultado do estresse crônico, típico do cotidiano do trabalho, principalmente quando, neste, existe excessiva pressão, conflitos e poucas recompensas emocionais (Harrison, 1999). De acordo com Gil-Monte (2005, 2008), a SB é uma resposta ao estresse laboral crônico, característica dos profissionais que trabalham diretamente atendendo pessoas e constitui-se de quatro dimensões assim caracterizadas: 1) ilusão pelo trabalho, avaliada de forma inversa para caracterizar burnout, é definida como a percepção de que o trabalho proporciona desafios e o 
alcance de metas profissionais é uma fonte de realização pessoal; 2) desgaste psíquico, caracterizado pelo sentimento de cansaço físico e emocional em ter de lidar, diariamente, em seu trabalho, com estressores e pessoas que apresentam algum tipo de problema; 3) indolência, entendida como a presença de atitudes negativas de indiferença, insensibilidade e distanciamento frente aos clientes, colegas e organização; 4) culpa, definida pelo surgimento de sentimentos de culpabilização por atitudes e comportamentos não condizentes com as normas internas e cobrança social acerca do papel profissional.

Pesquisas em diferentes culturas indicam que os professores estão entre os profissionais que apresentam maiores níveis de estresse no trabalho (Stoeber \& Rennert, 2008), sendo uma das atividades ocupacionais mais expostas a ambientes conflituosos e à alta exigência de trabalho (Araújo, Carvalho, Porto, Reis, \& Silvany Neto, 2005). A docência tem sido, nos últimos anos, uma das categorias profissionais mais investigadas nos estudos sobre burnout (Brouwers, Tomic, \& Boluijt, 2011). O crescente aumento se deve às importantes repercussões negativas na saúde física e mental do professor, no prejuízo da qualidade do processo de ensino-aprendizagem (Maslach \& Goldberg, 1998; Vaezi \& Fallah, 2011), no aumento de afastamentos do trabalho e pela tendência em abandonar a profissão (Rudow, 1999; Skaalvik \& Skaalvik, 2011), sendo essa repercussão muito preocupante, considerando que Freudenberger (1974), na fase inicial de estudos atinentes à burnout, advertia que a síndrome acomete profissionais altamente entusiasmados e socialmente comprometidos com o trabalho. Por conseguinte, a educação tende a perder trabalhadores identificados e comprometidos com o seu trabalho.

Burnout em professores é explicada pela alta exposição a estressores psicossociais (Carlotto, 2012), ampliada, principalmente, pelo processo de expansão das funções docentes (León, 2011; Noronha, Assunção \& Oliveira, 2008). No atual contexto, o professor deve, além de ministrar aulas atendendo às novas demandas, como o domínio de tecnologias, a abordagem de assuntos diversos, como saúde, sexualidade, meio ambiente, educação no trânsito, entre outros (Dias, 2011), também, executar trabalhos administrativos, organizar atividades extraescolares, participar de reuniões pedagógicas e de coordenação, seminários, conselhos de classe, atender necessidades/questões individuais relativas às dificuldades $\mathrm{da}$ aprendizagem de alunos, cuidar do patrimônio, material, recreios e locais de refeições (Nacarato, Varani \& Carvalho, 2000;
Schnetzler, 2000; Woods, 1999), tendo, assim, de ocupar tempo extra da sua carga horária atendendo pais (Skaalvik \& Skaalvik, 2009).

Apesar do aumento das demandas e investimentos, esses não se revertem em maior apoio e reconhecimento social. Pela perspectiva pública, a categoria de professores sofre muitas críticas, é extremamente cobrada em seus fracassos e, raramente, é reconhecida por seu sucesso (Farber, 1991, Vieira et al., 2010). Pelo exposto, verifica-se que o aprofundamento da intensificação do trabalho ocorre, principalmente, em dois aspectos: a ampliação das demandas profissionais, administrativas e burocráticas, e a autointensificação, pela exploração do sentimento de profissionalismo docente, e de suas autoimagens, focadas no cuidado e no zelo, fazendo com que o professor se sinta responsabilizado pela qualidade da educação e culpabilizado no caso de seu insucesso (Vieira et al., 2010). Essas questões ocasionam ao docente uma maior carga de trabalho, não somente em termos de volume, mas, igualmente, uma sobrecarga, cognitiva e emocional, sendo essa uma das variáveis comumente associadas ao burnout (Alarcon, 2011; Fernet, Austin, Trépanier \& Dussault, 2013; Maslach, Schaufeli \& Leiter, 2001; Vegchel, Jonge, Söderfeldt, Dormann \& Schaufeli, 2004; Yong \& Yue, 2007).

Embora já exista consenso acerca do maior poder explicativo desempenhado pelos estressores psicossociais de natureza ocupacional e organizacional, fatores individuais como características de personalidade, sistema de crenças, estratégias de coping e competências sociais e emocionais têm desempenhado um importante papel mediador entre os estressores na síndrome de burnout (Dias \& Queirós, 2010; González \& Garrosa, 2007). As pessoas que compreendem suas experiências emocionais sentem-se mais competentes para responder às demandas laborais (Extremera, Durán \& Rey, 2010).

Diversos estudos têm evidenciado uma relação negativa entre a autoeficácia e a síndrome de burnout (Aftab, Shah, \& Mehmood, 2012; Extremera, Durán, \& Rey, 2010; Fernet, Austin, Trépanier, \& Dussault, 2013; García-Renedo, Llorens, Cifre, \& Salanova, 2006; Vegchel, Jonge, Söderfeldt, Dormann, \& Schaufeli, 2004; Yong \& Yue, 2007). No entanto, poucos são os estudos que têm procurado identificar seu papel moderador entre fatores de risco e burnout (Salanova, Peiró \& Schaufeli, 2002). Cherniss (1993) refere que, independentemente da profissão, a autoeficácia desempenha importante papel no desenvolvimento de Burnout. 
Altas demandas e poucos recursos pessoais para execução do trabalho podem ocasionar sucessivas crises de autoeficácia, que, com o tempo, podem conduzir à burnout (Llorens, García-Renedo \& Salanova, 2005).

A autoeficácia, na perspectiva de Bandura (1977), é o conjunto de crenças que a pessoa tem sobre sua capacidade para organizar e executar ações necessárias para produzir determinadas metas. A crença otimista na própria competência para lidar com desafios diários aumenta a motivação para participar, de forma construtiva, frente às demandas da profissão. Em professores, essa questão é fundamental na medida em que as situações cotidianas, se avaliadas como desafios, contribuem para um melhor desempenho profissional $(\mathrm{Xu}$, 2012). A adaptação bem-sucedida às situações estressantes impede o surgimento de burnout (Aftab, Shah \& Mehmood, 2012; Brouwers, Tomic \& Boluijt, 2011; Schwerdtfeger, Konermann \& Schönhofen, 2008; Skaalvik \& Skaalvik, 2007). Professores com altos níveis de eficácia na gestão de sala de aula apresentam menores níveis de burnout, independentemente de variáveis como ambiente e suporte administrativo da sua escola (Durr, 2008; Mede, 2009).

Nessa perspectiva, esta pesquisa baseia-se no modelo apresentado na figura 1 , pelo qual a autoeficácia desempenha papel mediador na relação entre a sobrecarga e as dimensões de burnout.

Conforme o exposto, o presente estudo pretendeu avaliar se a autoeficácia poderia funcionar como variável mediadora na relação entre a sobrecarga de trabalho e as dimensões de burnout, para tanto, manejou as seguintes hipóteses:

H1: A autoeficácia desempenha um papel moderador na associação entre sobrecarga laboral e ilusão pelo trabalho.
H2: A autoeficácia desempenha um papel moderador na associação entre sobrecarga laboral e desgaste psicológico.

H3: A autoeficácia desempenha um papel moderador na associação entre sobrecarga laboral e indolência.

H4: A autoeficácia desempenha um papel moderador na associação entre sobrecarga laboral e culpa.

\section{Método}

\section{Participantes}

A amostra constituiu-se de 982 professores que exercem atividade docente em nove escolas públicas e seis escolas privadas localizadas na zona urbana de três cidades, na região metropolitana de Porto Alegre - RS. Todas podem ser caracterizadas como de médio porte, possuem os três níveis de ensino e equivalem-se quanto ao número de professores ( 35 a 80$)$ e de alunos (600 a 800). Foram excluídos os professores que exerciam somente atividades administrativas e os que estavam na instituição a menos de seis meses. Os professores investigados, em sua maioria, são mulheres $(83,8 \%)$, possuem união estável $(69,3 \%)$, têm filhos $(60,5 \%)$ e apresentam idade média de 39 anos $(D P=10,15)$. A quase totalidade dos professores tem formação em nível de graduação e pós-graduação (90,1\%). Possuem, em média. 13,6 anos de experiência profissional $(D P=9,34)$, trabalham, em média, 30 horas semanais $(D P=14,5)$ e contatam diariamente uma média de 101 alunos $(D P=95)$. Quanto ao tipo de escola, $64,4 \%$ de professores atuam, na maior parte de sua carga horária, em escola pública e trabalham somente em uma instituição $(65,6 \%)$.

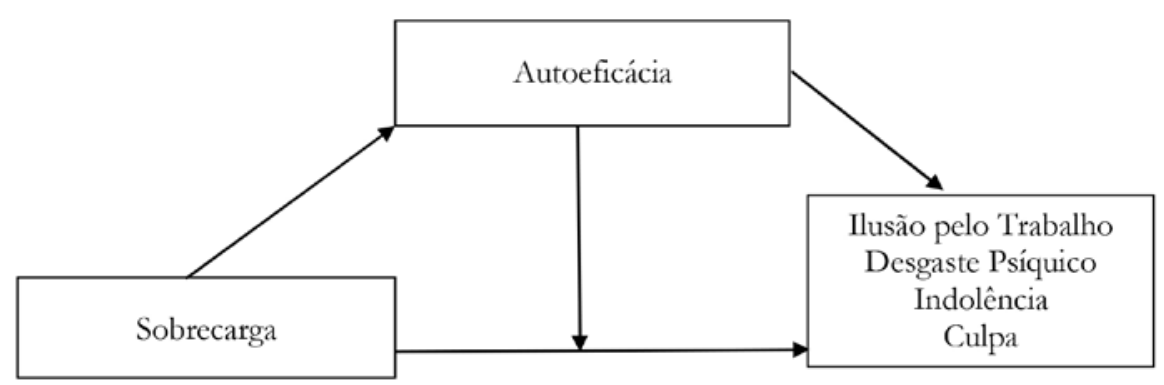

Figura 1. Representação gráfica do papel mediador da autoeficácia como variável mediadora entre a sobrecarga e as dimensões de Burnout. 


\section{Instrumentos}

Os dados foram levantados por meio dos seguintes instrumentos: 1. Questionário de dados sociodemográficos (sexo, idade, relacionamento conjugal, filhos, escolaridade) e laborais (tempo de experiência profissional, carga horária, número de alunos contatados diariamente, tipo de escola, trabalhar em outra instituição); 2. Questionário para avaliação da síndrome de burnout, versão para professores - Questionário para a Avaliação da Síndrome de Quemarse por el Trabajo - CESQT-PE (Gil-Monte, 2005), adaptado para o uso no Brasil por Gil-Monte, Carlotto e Câmara (2010). Este consta de vinte itens que se distribuem em quatro subescalas: ilusão pelo trabalho (5 itens, $\alpha=0,83$ ); desgaste psíquico ( 4 itens, $\alpha=0,80$ ), indolência ( 6 itens, $\alpha=0,80$ ) e culpa ( 5 itens, $\alpha=0,82$ ). Os itens são avaliados com uma escala tipo Likert, de frequência de cinco pontos (0 (nunca) a 4 (todos os dias)). 3. Escala geral de autoeficácia de Bäbler e Schwarzer (1996), adaptada por Gil-Monte (2005), constituída por oito itens $(\alpha=0,79)$. Todos os itens são avaliados com uma escala tipo Likert, de frequência de cinco pontos (0 (nunca) a 4 (todos os dias)). 4. Subescala de sobrecarga laboral da Organizational Stress Questionnaire - OSQ - (Caplan, Cobb, French, Van Harrison \& Pinneau, 1975), adaptada por Gil-Monte (2005), composta de oito itens $(\alpha=0,85)$. Todos os itens são avaliados com uma escala tipo Likert, de frequência de cinco pontos (0 (nunca) a 4 (todos os dias)).

\section{Procedimentos}

Primeiramente, foi realizado um contato com a direção de cada instituição de ensino, momento em que foi apresentado o objetivo do estudo a fim de obter a autorização e o apoio para a aplicação dos instrumentos. Estes foram entregues pessoalmente aos professores, sendo a coleta realizada ao final da aplicação. Foram realizados os procedimentos éticos conforme resolução 196 do Conselho Nacional de Saúde (CNS), assim, a pesquisa tem autorização do Comitê de Ética da Universidade Luterana do Brasil. Esclareceu-se aos professores e diretores das instituições de ensino que se tratava de uma pesquisa sem quaisquer efeitos avaliativos individuais e/ou institucionais e que as respostas seriam anônimas e confidenciais.

O banco de dados foi digitado e, posteriormente, analisado por meio do SPSS 17.0 (Statistical Package for the Social Sciences). Primeiramente, realizaram-se análises descritivas de caráter exploratório a fim de avaliar a distribuição dos itens, casos omissos ou possíveis erros de digitação. As variáveis sexo, idade e tempo de experiência profissional foram controladas em cada análise. $\mathrm{O}$ modelo proposto estabelece burnout como variável dependente (VD), considerando suas quatro dimensões - ilusão pelo trabalho, desgaste psicológico, indolência, culpa -, autoeficácia como variável mediadora (VM) e sobrecarga de trabalho como variável independente (VI). Com o objetivo de explorar as relações entre as variáveis, foram realizadas análises de regressão múltipla hierárquica para verificar a relação preditiva da sobrecarga de trabalho nas dimensões do burnout e, ainda, para identificar se a autoeficácia funciona como mediadora dessas relações.

Todos os pressupostos de normalidade, multicolinearidade, linearidade e homocestacidade foram testados, não tendo sido identificadas violações de acordo com orientações de Tabachnick e Fidell (2001). Para que seja afirmado que uma variável é mediadora, Baron e Kenny (1986) explicitam três condições: o mediador é preditor significativo da variável dependente; a variável independente é um preditor significativo do mediador; e, na presença da variável independente e do mediador, a relação significativa que existia entre a variável independente e a dependente decresce em magnitude.

O tratamento dos dados obedeceu a um nível de confiança de 95\%, com um nível de significância de $5 \%$ (valor de $\mathrm{p} \leq 0,05$ ). No entanto, é importante o uso de métodos alternativos para a análise de dados nas investigações, em particular, no âmbito das ciências sociais, nomeadamente, devido às críticas crescentes do uso da estatística inferencial. Nesse sentido, adiciona-se, na apresentação dos resultados deste estudo, o tamanho do efeito (effect size). Assim, quanto maior for o tamanho do efeito, maior será a manifestação do fenômeno em estudo na população. Em termos práticos, essa medida codifica a informação quantitativa crítica encontrada nos estudos, tentando dar resposta à significância prática dos resultados, seja clínica ou educacional (Conboy, 2003). Na análise de regressão, o tamanho do efeito foi obtido por meio dos coeficientes de regressão padronizados, calculados para cada modelo (Field, 2009).

\section{Resultados}

\section{Análises descritivas}

$\mathrm{Na}$ Tabela 1, são apresentas as médias, o desvio padrão, valores de alfa e matriz de correlação entre as variáveis em estudo. Resultados evidenciam maior média 
na dimensão de burnout de "ilusão pelo trabalho" e a menor média em "indolência". Os instrumentos revelam índices adequados de consistência interna avaliados pelo $\alpha$ de Cronbach (Bland \& Altman, 1997), variando de 0,72 a 0,88 . A variáveis apresentam correlações que se alteram de fracas $(r=0,16)$ a moderadas $(r=0,60)$.

\section{Análises de regressão avaliando o papel mediador da autoeficácia}

Os resultados apresentados referem-se às análises de regressão múltipla entre a sobrecarga laboral (variável independente) e as dimensões do burnout (variável dependente). Em seguida, são apresentadas as análises referentes às três condições de Baron e Kenny (1986) para identificar a relação de mediação da autoeficácia nas dimensões do burnout.

Na Tabela 2, expõe-se o resultado da regressão linear entre a sobrecarga laboral e as dimensões do burnout. Os resultados evidenciam que a sobrecarga laboral prediz significativamente, de forma positiva, as dimensões "desgaste psicológico", "indolência" e "culpa"; e de forma negativa, a dimensão "ilusão pelo trabalho", explicando 18\% da dimensão, com um tamanho do efeito que varia de pequeno $(0,047)$ a médio $(0,181)$.

Quanto ao teste de mediação, foram testadas as três condições citadas por Baron e Kenny (1986). A primeira delas afirma que a variável mediadora (autoeficácia) deve ser preditora da variável dependente (dimensões do burnout), condição essa que foi confirmada pelos resultados obtidos (ilusão: $\mathrm{R}^{2}=0,152$, beta $=0,390$, $p=0,000$; desgaste psicológico: $\mathrm{R}^{2}=0,078$, Beta $=-0,279$, $p=0,000)$; indolência: $\mathrm{R}^{2}=0,088$, beta $=-0,297, p=0,000$; culpa: $\mathrm{R}^{2}=0,027$, beta $=-0,165, p=0,000$ ).

Para a segunda condição, é necessário que a variável independente (sobrecarga laboral) prediga a variável mediadora (autoeficácia). O teste de regressão indica que essa condição é cumprida, em que a sobrecarga laboral prediz de forma negativa a autoeficácia $\left(\mathrm{R}^{2}=0,026\right.$, beta $\left.=-0,162, p=0,000\right)$.

Por fim, a terceira condição afirma que, na presença da variável independente e do mediador, a relação

Tabela 1. Estatística Descritiva, Valores de Alfa e Correlações entre as Variáveis em Estudo

\begin{tabular}{|c|c|c|c|c|c|c|c|c|}
\hline Variáveis & $\mathrm{M}$ & DP & $\alpha$ & 1 & 2 & 3 & 4 & 5 \\
\hline 1. IL & 2,90 & 0,84 & 0,79 & 1 & & & & \\
\hline 2. DP & 1,56 & 0,89 & 0,79 & $-0,30^{* *}$ & 1 & & & \\
\hline 3. IN & 1,06 & 0,74 & 0,77 & $-0,47^{* *}$ & $0,49^{* *}$ & 1 & & \\
\hline 4. CL & 1,17 & 0,79 & 0,78 & $-0,26^{* *}$ & $0,34^{* *}$ & $0,60^{* *}$ & 1 & \\
\hline 5. Sobrecarga & 1,76 & 0,64 & 0,72 & $-0,22^{* *}$ & $0,42^{* *}$ & $0,40^{* *}$ & $0,42^{* *}$ & 1 \\
\hline 6. Autoeficácia & 2,92 & 0,61 & 0,88 & $0,39^{* *}$ & $-0,28^{* *}$ & $-0,30^{* *}$ & $-0,16^{* *}$ & $-0,16^{* *}$ \\
\hline
\end{tabular}

Nota: $* * p<0,01$.

Tabela 2. Regressão Linear entre a Sobrecarga Laboral e as Dimensões do Burnout

\begin{tabular}{|c|c|c|c|c|c|}
\hline Variáveis & $\mathrm{R}$ & $\mathrm{R}^{2}$ & Beta & $\mathrm{t}$ & $\mathrm{p}$ \\
\hline \multicolumn{6}{|l|}{ Ilusão } \\
\hline Sobrecarga Laboral & 0,216 & 0,047 & $-0,216$ & $-6,907$ & $0,000^{* *}$ \\
\hline \multicolumn{6}{|l|}{ Desgaste psicológico } \\
\hline Sobrecarga Laboral & 0,423 & 0,179 & 0,423 & 14,581 & $0,000 * *$ \\
\hline \multicolumn{6}{|l|}{ Indolência } \\
\hline Sobrecarga Laboral & 0,402 & 0,161 & 0,402 & 13,724 & $0,000^{* *}$ \\
\hline \multicolumn{6}{|l|}{ Culpa } \\
\hline Sobrecarga Laboral & 0,425 & 0,181 & 0,425 & 14,696 & $0,000^{* *}$ \\
\hline
\end{tabular}

$* * \mathrm{p} \leq 0,01$. 
significativa que existia entre a variável independente e a dependente decresce em magnitude. A Tabela 3 apresenta o teste de mediação para a variável independente sobrecarga laboral. Os resultados indicam que, na presença da variável autoeficacia, a relação entre a sobrecarga laboral e as dimensões do burnout decrescem em magnitude (beta diminui) e o poder explicativo das dimensões do burnout aumenta ( $\mathrm{R}^{2}$ aumenta), ou seja, há relação de mediação.

Uma síntese dos resultados é apresentada na Figura 2, cujas linhas contínuas representam relações positivas, e as linhas interrompidas, as relações negativas. Todas as relações descritas são significativas $(\mathrm{p} \leq$ $0,05)$.

Tabela 3. Relaçôes entre a Variável Independente (Sobrecarga Laboral), a Mediadora (Autoeficácia) e a Dependente (Dimensões do Burnout)

\begin{tabular}{|c|c|c|c|c|c|}
\hline Variáveis independentes e medidora & Variável Dependente & $\mathrm{R}^{2}$ & Beta & $\mathrm{F}$ & $\mathrm{p}$ \\
\hline Sobrecarga laboral $^{a}$ & Ilusão pelo trabalho & 0,047 & $-0,216$ & $44,829 * *$ & 0,000 \\
\hline Sobrecarga laboral ${ }^{a}$ & & 0,047 & $-0,157$ & $98,073^{* *}$ & 0,000 \\
\hline Autoeficácia ${ }^{\text {b }}$ & & 0,176 & 0,365 & & 0,000 \\
\hline Sobrecarga laboral $^{a}$ & Desgaste psicológico & 0,179 & 0,423 & $199,773 * *$ & 0,000 \\
\hline Sobrecarga laboral ${ }^{a}$ & & 0,179 & 0,388 & $132,721 * *$ & 0,000 \\
\hline Autoeficácia b & & 0,224 & $-0,217$ & & 0,000 \\
\hline Sobrecarga laboral ${ }^{a}$ & Indolência & 0,161 & 0,402 & $176,995^{* *}$ & 0,000 \\
\hline Sobrecarga laboral ${ }^{a}$ & & 0,161 & 0,363 & $127,083^{* *}$ & 0,000 \\
\hline Autoeficácia ${ }^{\text {b }}$ & & 0,217 & $-0,238$ & & 0,000 \\
\hline Sobrecarga laboral ${ }^{a}$ & Culpa & 0,181 & 0,425 & $202,946^{* *}$ & 0,000 \\
\hline Sobrecarga laboral ${ }^{a}$ & & 0,181 & 0,409 & $107,999 * *$ & 0,000 \\
\hline Autoeficácia ${ }^{b}$ & & 0,190 & $-0,099$ & & 0,001 \\
\hline
\end{tabular}

Nota: ${ }^{a}$ variável independente; ${ }^{\mathrm{b}}$ variável mediadora; ${ }^{* *} \mathrm{p} \leq 0,01$.

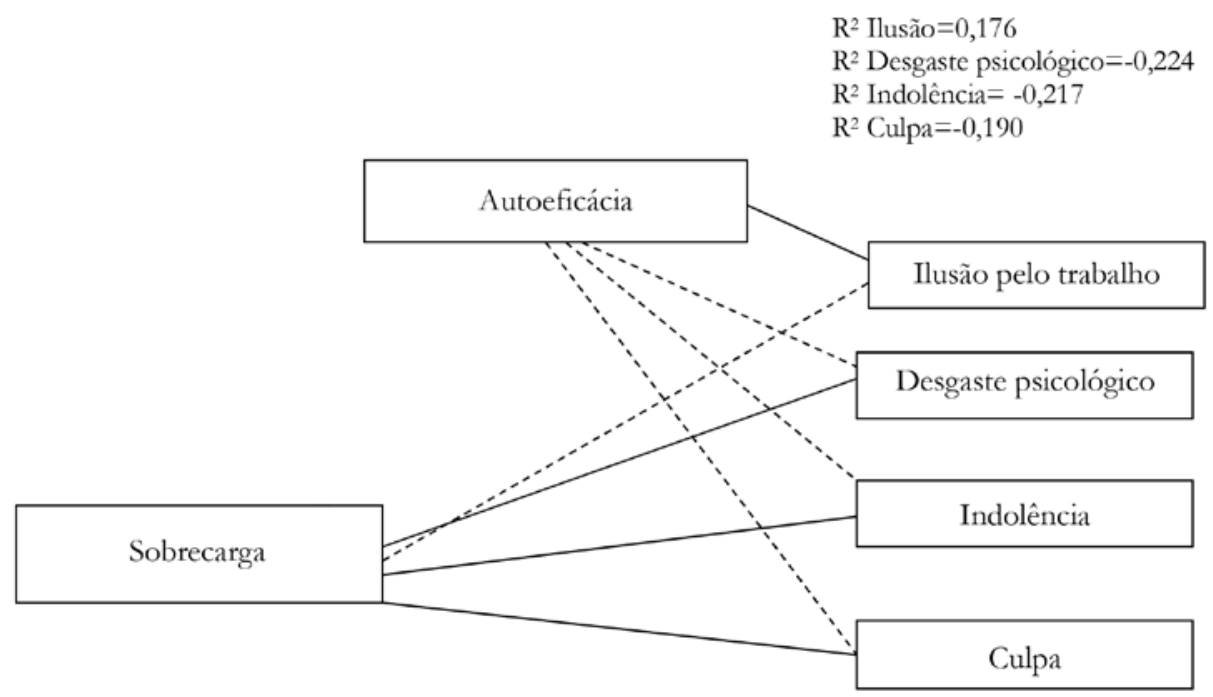

Figura 2. Relações entre a variável independente (sobrecarga laboral), a mediadora (autoeficácia) e a dependente (dimensões do burnout) 


\section{Discussão}

O objetivo do presente estudo foi avaliar se a autoeficácia poderia funcionar como variável mediadora na relação entre a sobrecarga de trabalho e as dimensões de burnout. Os resultados obtidos confirmam as quatro hipóteses do estudo, em que a autoeficácia desempenha um papel mediador entre a sobrecarga e as dimensões de burnout. Esse resultado é consistente com os estudos que identificaram o papel mediador da autoeficácia entre estressores psicossociais e a SB (Llorens, García-Renedo, \& Salanova, 2005; Salanova, Peiró, \& Schaufeli, 2002; Schwarzer \& Hallum, 2008; Van Dick \& Wagner, 2001). Esse achado pode ser explicado, segundo Bandura (1997), pela relação positiva que a autoeficácia apresenta com o sentimento de realização e bem-estar pessoal. Pessoas que acreditam na sua capacidade e lidam com tarefas difíceis, vendo-as como desafios a serem manejados e controlados, administram de forma adequada os estressores. No caso do presente estudo, pode-se pensar que os professores com maior autoeficácia conseguem atenuar o impacto da sobrecarga laboral derivada das inúmeras atividades presentes na atual profissão docente sobre as dimensões do burnout.

Especificamente, com relação à dimensão de ilusão pelo trabalho, uma possível explicação do resultado, que indica que o sentimento de autoeficácia modera o impacto da sobrecarga, é que professores com alto sentimento de eficácia persistem e mantêm-se motivados e entusiasmados nas tarefas empreendidas, criam um contexto de aula favorável e, consequentemente, conseguem melhores resultados (Fernández-Arata, 2008; Mansfield, Wosnitza, \& Beltman, 2012; Shaughnessy, 2004; Tschannen-Moran \& Hoy, 2001). A autoeficácia em professores influencia seu comportamento em auxiliar o aluno para realizações e aprendizagens, assim como a orientação para seu próprio desenvolvimento e nível de satisfação profissional (Pedro, 2011). Bandura (1997) afirma que a autoeficácia motiva os indivíduos, dessa maneira, determinando a escolha dos desafios que eles optam por empreender, fazendo com que o estabelecimento de metas pessoais seja influenciado pela autoavaliação de suas capacidades. A autoeficácia influencia as metas e aspirações de carreira de uma forma positiva (Garcia, 2009).

O desgaste psicológico é mediado pela autoeficácia para o estressor sobrecarga, desse modo, confirmando estudo realizado por Salanova, Peiró e Schaufeli (2002). Pode-se entender que os docentes que acreditam na sua capacidade de lidar com as inúmeras demandas profissionais apresentam menor desgaste psicológico, uma vez que tendem a interpretar tais situações como desafios ao invés de ameaças. A adaptação bem-sucedida às situações estressantes impede $\mathrm{O}$ surgimento da SB (Brouwers, Tomic, \& Boluijt, 2011; Schwerdtfeger, Konermann, \& Schönhofen, 2008; Skaalvik \& Skaalvik, 2007).

No que diz respeito à indolência, dimensão com foco nas relações interpessoais, verifica-se que a autoeficácia atenua sua relação com a sobrecarga. Frente às inúmeras atividades presentes no atual contexto docente, o ponto central do empreendimento desses profissionais reside nas relações estabelecidas não somente com o aluno, mas também com colegas, equipe e pais. Embora a relação com os alunos tenha um potencial gratificante ou estressante, a relação com os pais é de parceria, com implicações importantes na autoeficácia docente. Estudo de Skaalvik e Skaalvik (2010) revelou relação entre a dimensão de despersonalização de burnout e autoeficácia em professores. Os autores referem que a relação professor-pais pode afetar as atitudes dos alunos em relação à escola, assim como seus professores. A relação positiva entre professores e pais pode, portanto, ser um determinante importante da motivação do aluno para a realização de trabalhos escolares e adequação do seu comportamento na escola, dessa maneira, facilitando o trabalho docente. Os autores destacam que os pais têm participado de forma mais ativa na escola, fazendo exigências sobre os métodos e os conteúdos do ensino. Nesse sentido, a qualidade da relação estabelecida entre os professores e os pais evidencia-se como fundamental para evitar ou diminuir a dimensão de despersonalização.

O impacto da sobrecarga de trabalho sobre a dimensão de culpa é mediado pela autoeficácia, conduzindo a uma possível explicação referida por Hochwarter, Perrewe, James Meurs e Kacmar (2007). Segundo os autores, a percepção da capacidade de administrar os recursos individuais no trabalho pode ser vista como uma forma de controle pessoal, que ajuda a reduzir os efeitos negativos dos sentimentos de culpa relacionados com o trabalho. A culpa desempenha uma função pró-social no fortalecimento das relações (Etxebarría, 2000; Tangney, Wagner, \& Gramzow, 1992), levando as pessoas a uma reconsideração acerca de seu próprio comportamento (Baumeister, Stiwell, \& Heatherton, 1995). No contexto docente, pode-se pensar que o professor, ao conhecer suas capacidades 
e competências, consiga manejar de forma positiva as demandas laborais, diminuindo o sentimento de culpa na medida em que consegue realizar uma análise mais precisa acerca da cobrança social e limitações de seu papel profissional.

\section{Conclusão}

Os resultados revelam a importância da autoeficácia como variável que pode atenuar o impacto da sobrecarga laboral sobre as dimensões de burnout, com isso, auxiliando na elucidação de um dos possíveis mecanismos que pode proteger os professores deste tipo de adoecimento ocupacional. Nesse sentido, desenvolver a autoeficácia do professor, possivelmente, seja um importante recurso para impedir, não só o desenvolvimento da síndrome, mas, também, para melhorar seu desempenho e manter-se na profissão (Costas, Carsona, Matthewsb, Grawitchc, \& Barberd, 2010). Ainda, é importante ressaltar que a realidade é, provavelmente, muito mais complexa, e que muitas outras variáveis individuais podem desempenhar um relevante papel mediador entre a sobrecarga e a SB, como, por exemplo, variáveis de personalidade e estratégias de coping.

Deve-se ter cautela com relação aos resultados obtidos, pois são decorrentes de uma amostra não probabilística de instituições localizadas em uma região específica do país, não sendo, portanto, passíveis de generalizações para outras instituições ou profissionais. Novos estudos devem ser realizados no contexto brasileiro com amostras probabilísticas e com professores de diferentes regiões e culturas escolares. Sugerem-se, também, estudos de delineamento longitudinal porque o desenvolvimento da autoeficácia possui relação com o tempo de experiência profissional (Woolfolk Hoy \& Burke Spero, 2005).

\section{Referências}

Alarcon, G. M. (2011). A meta-analysis of burnout with job demands, resources, and attitudes. Journal of Vocational Behavior, 79(2), 549-562.

Aftab, N., Shah, A. A., \& Mehmood, R. (2012). Relationship of self efficacy and burnout among physicians. Academic Research International, 2(2), 539-548.

Araújo, T. M., Carvalho, F. M., Porto, L. A., Reis, E. J. F. B., \& Silvany Neto, A. M. (2005). Trabalho e distúrbios psíquicos em professores da rede municipal de Vitória da Conquista, Bahia, Brasil. Cadernos de Saúde Pública, 21(5), 1480-1490.

Bandura, A. (1997). Self-efficacy: The exercise of control. New York: Freeman.

Baron, R. M., \& Kenny, D. A. (1986). The moderatormediator variable distinction in social psychological research: conceptual, strategic, and statistical considerations. Journal of Personality and Social Psychology, 51, 1173-1182.

Baumeister, R. F., Stillwell, A. M., \& Heatherton, T. F. (1994). Guilt: An interpersonal approach. Psychological Bulletin, 115(2), 243-267.

Bland J., \& Altman D. (1997) Statistics notes: Cronbach's alpha. British Medical Journal, 314, 570-572.

Brouwers, A., Tomic, W., \& Boluijt, H. (2011). Job demands, job control, social support and self-efficacy beliefs as determinants of burnout among physical education teachers. Europe's Journal of Psychology, 1, 17-39.

Carlotto, M. S. (2012). Sindrome de burnout em professores: Avaliação, fatores associados e intervenção. Porto: LivPsic.

Cherniss, C. (1993). The role of professional self-efficacy in the etiology and amelioration of burnout. In W. B. Schaufeli, C. Maslach \& T. Marek (Eds.), Professional burnout: recent development in theory and research (pp. 135-149). Washington;: Taylor \& Francis.

Cole, D., Panchanadeswaran, S., \& Daining, C. (2004). Predictors of job satisfaction of licensed socialworkers. Journal of Social Service Research, 31(1), 1-12.

Costas, N. T., Carsona, R. L., Matthewsb, R., Grawitchc, M. J., \&. Barberd, L. K. (2010). Exploring the association between teacher's perceived student misbehaviour and emotional exhaustion: the importance of teacher efficacy beliefs and emotion regulation. Educational Psychology, 30(2), 173-189.

Dias, S., \& Queirós, C. (2010). A influência dos traços de personalidade no burnout dos professores. In C. Nogueira, I. Silva, L. Lima, A. T. Almeida, R. Cabecinhas, R. Gomes, C. Machado, A. Maia, A. Sampaio \& M. C. Taveira (Eds.), Actas do VII Simpósio Nacional de Investigação em Psicologia (pp.1066-1080). Retirado de http://www.actassnip2010.com; 
Dias, S. de F. (2011). Construção da identidade docente: intermediações da formação e das condições de trabalho do professor. Revista Educação, 6(1), 45-53.

Durr, J. A. (2008). Identifying teacher capacities that may buffer against teacher burnout. Theses of Doctor of Philosophy. Ohio State University. EUA.

Etxebarría, I. (2000). Guilt: an emotion under suspicion. Psicothema, 12(Supl), 101-108.

Fernet, C., Austin, S., Trépanier, S-G., \& Dussault, M. (2013). How do job characteristics contribute to burnout? Exploring the distinct mediating roles of perceived autonomy, competence, and relatedness. European Journal of Work and Organizational Psychology, 22(2), 123- 137.

Field, A. (2009) Discovering Statistics with SPSS ( $\left.2^{\mathrm{a}} \mathrm{ed}.\right)$. London: Sage Publications Ltd.

Freudenberger, H. J. (1974). Staff burnout. Journal of Social Issues, 30, 159-165.

García-Renedo, M., Llorens, S., Cifre, E., \& Salanova, M. (2006). Antecedentes afectivos de la auto-eficacia docente: un modelo de relaciones estructurales. Revista de Educación, 339, 387-400.

Gil-Monte, P. R. (2005) El sindrome de quemarse por el trabajo (burnout). Una enfermidad laboral en la sociedad del bienestar. Pirâmide, Madrid.

Gil-Monte, P. R. (2008). El síndrome de quemarse por el trabajo (burnout) como fenómeno transcultural. Informació Psicológica, 91-92, 4-11.

Gil-Monte, P. R., Carlotto, M. S., \& Câmara, S. (2010). Validation of the Brazilian version of the Spanish burnout Inventory in teachers. Revista de Saúde Pública, 44(1), 140-147.

González, J. L., \& Garrosa, E. (2007). Líneas y estrategias de actuación focalizadas en el individuo: propósitos específicos. En P. R. Gil-Monte \& B. Moreno-Giménez (Eds.), El sindrome de quemarse por el trabajo (burnout). Grupos profesionales de riesgo. Madrid: Pirámide.

Guglielmi, R. S., \& Tatrow, K. (1998). Occupational stress, burnout, and health in teachers: a methodological and theoretical analysis. Review of Educational Research, 68(1), 61-69.

Harrison, B. J. (1999). Are you to burn out? Fund Raising Management, 30(3), 25-28.
Hochwarter, W. A., Perrewe, P. L., Meurs, J. A., \& Kacmar, C. (2007). The interactive effects of work-induced guilt and ability to manage resources on job and life satisfaction. Journal of Occupational Health Psychology, 12(2), 125-135.

International Labour Office (2012). Stress prevention at work checkpoints: Practical improvements for stress prevention in the workplace Geneva: International Labour Office.

Klassen, R. M., \& Chiu, M. M. (2010). Effects on teacher's self-efficacy and job satisfaction: Teacher gender, years of experience, and job stress. Journal of Educational Psychology, 102(3), 741-756.

Llorens, S., García, M., \& Salanova, M. (2005). burnout como consecuencia de una crisis de eficacia: un estudio longitudinal en profesores de secundaria. Revista de Psicología del Trabajo y de las Organizaciones, 21, 55-70.

Mansfield, C., Wosnitza, M., \& Beltman, S. (2012). Goals for teaching: Towards a framework for examining motivation of graduating teachers. Australian Journal of Educational \& Developmental Psychology, 12, 21-34.

Maslach C., Schaufeli W. B., \&. Leiter M. P. (2001). Job Burnout. Annual Review of Psychology, 52, 397-422.

Maslach, C., \& Goldberg, J. (1998). Prevention of burnout: News perspectives. Applied \& Preventive Psychology, 7, 63-74.

Mazur, P. J., \& Lynch, M. D. (1989). Differential impact of administrative, organizational, and personality factors on teacher burnout. Teaching and Teacher Education, 5, 337-353.

Mede, E. (2009). An analysis of relations among personal variables, perceived self-efficacy and social support on burnout among Turkish EFL Teachers. Inonu University Journal of the Faculty of Education, 10(2), 39-52.

Nacarato, A. M., Varani, A., \& Carvalho, V. (2000). O cotidiano do trabalho docente: palco, bastidores e trabalho invisível abrindo as cortinas. Em C. M. G. Geraldi, D. Fiorentina \& E. M. A. Pereira. (Orgs.), Cartografias do trabalho docente (pp. 73-104). Campinas: Mercado de Letras.

Noronha, M. M. B., Assunção, A. A., \& Oliveira, D. A. (2008). O sofrimento no trabalho docente: $\mathrm{O}$ caso das professoras da rede pública de Montes 
Claros, Minas Gerais. Trabalho, Educação e Saúde, 6(1), 65-85.

Pedro, N. (2001). Autoeficácia e satisfação profissional dos professores: colocando os construtos em relação num grupo de professores do ensino básico e secundário. Revista de Educação, XVIII (1), 23-47.

Rudow, B. (1999). Stress and burnout in the teaching profession: European studies, issues, and research perspectives. In R. Vanderbergue \& M. A. Huberman (Eds.), Understanding and preventing teacher burnout: A source book of international practice and research (pp. 38-58). Cambridge: Cambridge University Press.

Salanova, M., Peiró, J. M., \& Schaufeli, W. B. (2002). Self efficacy specificity and Burnout among information technology workers: An extension of the job demands control model. European Journal of Work and Organizational Psychology, 11(1), 1-25.

Schnetzler, R. P. (2000). Prefácio. Em C. M. G. Geraldi, D. Fiorentina, \& E. M. de A. Pereira (Orgs.), Cartografias do trabalho docente (pp. 7-9). Campinas: Mercado de Letras.

Schwerdtfeger, A., Konermann, L., \& Schönhofen, K. (2008). Self-efficacy as a health-protective resource in teachers? A biopsychological approach. Health Psychology, 27(3), 358-368.

Schwarzer, R., \& Hallum, S. (2008). Perceived teacher self-efficacy as a predictor of job stress and burnout: mediation analyses. Applied Psychology: An International Review, 57, 152-171.

Skaalvik, E. M., \& Skaalvik, S. (2007). Dimensions of teacher self-efficacy and relations with strain factors, perceived collective teacher efficacy, and teacher burnout. Journal of Educational Psychology, 99, 611-625.

Skaalvik, S., \& Skaalvik, E. M. (2009). Job satisfaction and strains among teachers. A qualitative study. Bedre Skole, 2, 35-43.

Skaalvik, E. M., \& Skaalvik, S. (2011). Teacher job satisfaction and motivation to leave the teaching profession: Relations with school context, feeling of belonging, and emotional exhaustion. Teaching anda Teacher Education, 27(6), 1029-1038.

Stoeber, J., \& Rennert, D. (2008). Perfectionism in school teachers: Relations with stress appraisals, coping styles, and burnout. Anxiety, Stress \& Coping, 21, 37-53.

Tabachnick, B. G., \& Fidell, L. S. (2001). Using multivariate statistics ( $4^{a}$ ed.). Needham Heights, MA: Allyn \& Bacon.

Tangney, J. P., Wagner, P., \& Gramzow, R. (1992). Proneness to shame, proneness to guilt, and psychopathology. Journal of Abnormal Psychology, 101(3), 469-478.

Tschannen-Moran, M., \& Hoy, A. W. (2001). Teacher efficacy: Capturing an elusive construct. Teaching and Teacher Education, 17, 783-805.

Vaezi, S., \& Fallah, N. (2011).The Relationship between emotional intelligence and Burnout among Iranian EFL teachers. Journal of Language Teaching and Research, 2(5), 1122-1129.

Van Dick, R., \& Wagner, U. (2001).Stress and strain in teaching: A structural equation approach. British Journal of Educational Psychology, 81(2) 243-259.

Vegchel, van N., Jonge, de J., Söderfeldt, M., Dormann, C., \& Schaufeli, W. (2004). Quantitative versus emotional demands among Swedish human service employees: Moderating effects of job control and social support. International Journal of Stress Management, 11(1), 21-40.

Yong, Z., \& Yue, Y. (2007). Causes for burnout among secondary and elementary school teachers and preventive strategies. Chinese Education and Society, 40(5), 78-85.

$\mathrm{Xu}, \mathrm{L}$. (2012).The role of teacher's beliefs in the language teaching-learning process. Theory and Practice in Language Studies, 2(7), 1397-1402.

Recebido em: 08/10/2013 Primeira Reformulação em: 19/02/2014 Aprovado em: 15/10/2014 
Sobre as autoras:

Mary Sandra Carlotto é psicóloga, mestre em Saúde Coletiva (ULBRA-RS), doutora em Psicologia Social (USC/ES), professora do PPG em Psicologia e do PPG em Educação (PUCRS), coordenadora do grupo de pesquisa em Psicologia da Saúde Ocupacional (PUCRS) e bolsista produtividade do CNPq.

Sofia Raquel da Silva Dias é psicóloga, mestre na área da Educação para a Saúde (FM-FPCEUC), doutora em Psicologia (FPCEUP), técnica superior de Segurança no Trabalho, membro colaborador do CEFH (UCP), formadora do IEFP e enfermeira do CHVN Gaia/Espinho.

Jaqueline Brito Vidal Batista é psicóloga, mestre em Psicologia Social (UFPB), doutora em Saúde Pública (FIOCRUZ), professora do PPG em Enfermagem (UFPB/CCS) e de Psicologia da Educação (UFPB/CE).

Liciane Diehl é psicóloga, mestre em Psicologia (PUCRS) e professora do Centro Universitário UNIVATES.

Contato com as autoras:

Pontifícia Universidade Católica do Rio Grande do Sul - PUCRS

Programa de Pós-Graduação em Psicologia - Faculdade de Psicologia

Av. Ipiranga, 6681 - Prédio 11 - Sala 933 - Porto Alegre/RS

CEP: 90619-900

E-mail: mary.sandra@pucrs.br 